NISSUNA UMANA INVESTIGAZIONE SI PUO DIMANDARE VERA SCIENZIA S'ESSA NON PASSA PER LE MATEMATICHE DIMOSTRAZIONI LEONARDO DA VINCI

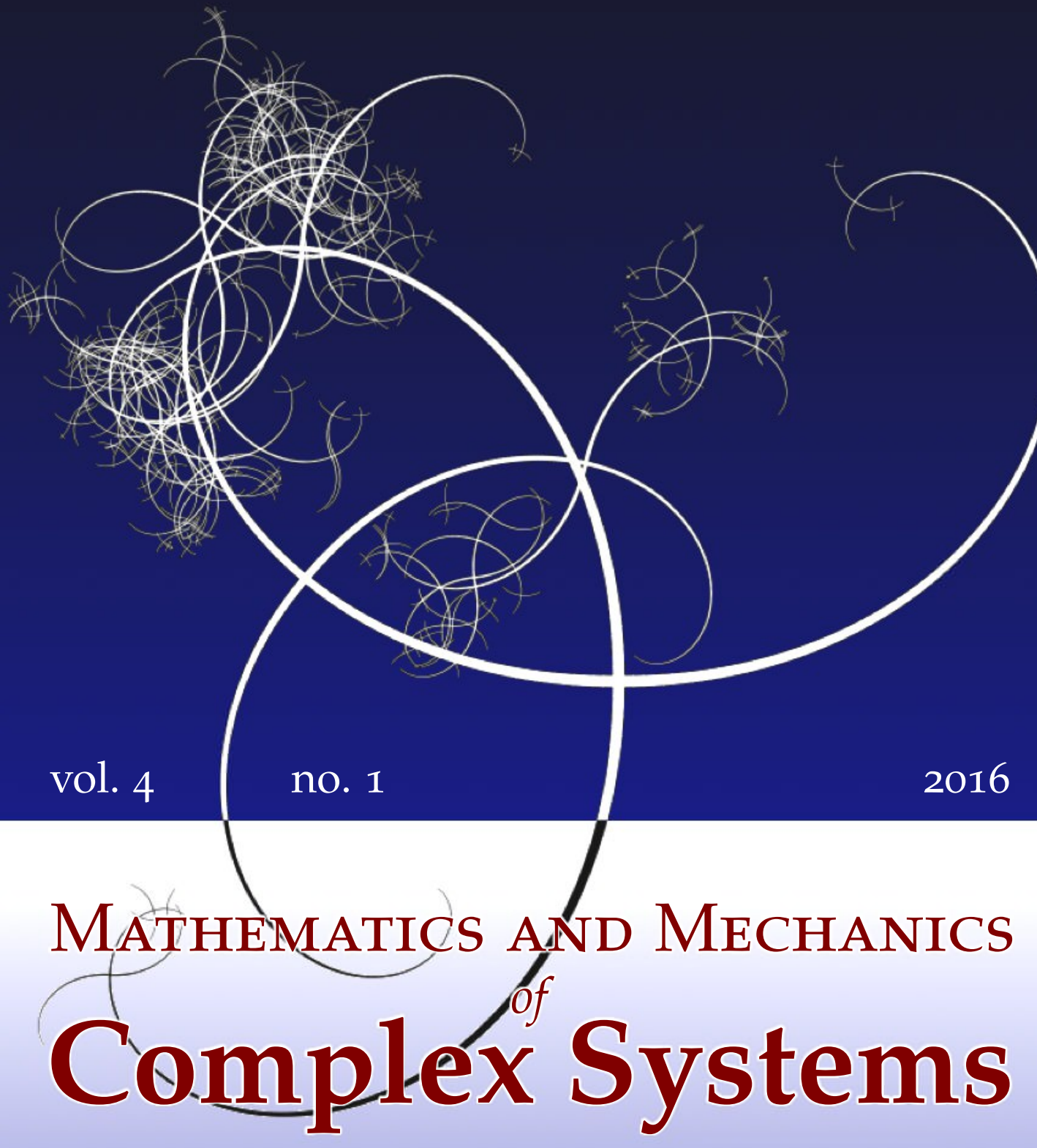

MARCElo EPSTEIN AND MANUEl DE LEÓN UNIFIED GEOMETRIC FORMULATION OF MATERIAL UNIFORMITY AND EVOLUTION 


\title{
UNIFIED GEOMETRIC FORMULATION OF MATERIAL UNIFORMITY AND EVOLUTION
}

\author{
Marcelo Epstein AND MANUel De LeÓN
}

\section{Dedicated to Gérard Maugin, in gratitude for many years of inspiration and friendship}

\begin{abstract}
The differential-geometric underpinnings of a unified theory of material uniformity and evolution are exposed in terms of the language of groupoids subordinate to geometric distributions. Both the standard theory of material uniformity and the extended theory of functionally graded materials are included in the formulation as well as their temporal counterparts in anelastic and aging processes.
\end{abstract}

\section{Introduction}

The delightful classical survey article by Alan Weinstein [1996] brings home the idea that groups, considered as the main carriers of information about the symmetries of a physical system, are actually not sufficient to convey the generality of the intuitive concept of symmetry. His example of the regular rectangular tiling of a bathroom floor, as opposed to the tiling of the whole plane, is very suggestive. Indeed, in passing from the infinite to the finite extent, while the symmetries of the individual tiles are preserved, the translational symmetries are lost. Nevertheless, any observer of the bathroom floor will agree that it still has a remnant of this distant kind of symmetry. In continuum mechanics, if we replace the bathroom floor with a material body $\mathcal{B}$, each tile can be identified with the tangent space $T_{X} \mathcal{B}$ at each point $X \in \mathcal{B}$. Any given constitutive equation at $X$ will enjoy some material symmetries, which are encoded in the material symmetry group $\mathcal{G}_{X}$ at $X$. But consider now another point $Y \in \mathcal{B}$. What would the meaning be of a distant symmetry between $X$ and $Y$ ? The clearest answer to this question is that $X$ and $Y$ are thus related if they are made of the same material. In the terminology of Walter Noll [1967/68], the points are materially isomorphic. A body all of whose points are materially isomorphic is said to be materially uniform.

\section{Communicated by Raffaele Esposito.}

The financial support of the Natural Sciences and Engineering Research Council of Canada and of the Consejo Superior de Investigaciones Científicas of Spain is gratefully acknowledged.

MSC2010: 74A20.

Keywords: groupoids, distributions, material uniformity, material evolution, body-time manifold, groupoid actions, aging, morphogenesis. 
In a philosophical sense, however, the notion of symmetry is, literally, in the eyes of the beholder. We may choose to declare the presence of a distant symmetry between two points if they are made of possibly different materials while enjoying the same symmetry type, ${ }^{1}$ a much weaker condition. In this case, the symmetry groups $\mathcal{G}_{X}$ and $\mathcal{G}_{Y}$ need only be conjugate. We call the equivalence relation associated with this property unisymmetry [Epstein and de León 2000]. Unisymmetric bodies are known in engineering applications as functionally graded materials, usually made of two components with a spatially varying composition.

Fixing attention on a particular material point and following its material response in time, we obtain the notion of material evolution [Epstein and Elżanowski 2007]. If, as time goes on, the material response remains materially isomorphic to its initial response, we have a case of pure remodeling or anelastic evolution [Wang and Bloom 1973/74]. The material in an infinitesimal neighbourhood undergoes a process of reaccommodation, but does not experience any other essential changes. This is clearly another (timewise) manifestation of the concept of distant symmetry. Any other change of material behaviour can be considered as a process of material aging. A particular case is obtained when, while the material ages, the symmetry type is preserved. In other words, the material evolves unisymmetrically. Finally, any change in the symmetry type gives rise to a process of morphogenesis [Turing 1952].

When the spatial and temporal distant symmetries are combined, it is reasonable to expect that the corresponding geometrical descriptor will be a properly defined material groupoid based on the consideration of a body-time manifold, which is the main object of consideration in the present article. Some basic definitions pertaining to the theory of groupoids and their actions on sets are reviewed in Section 2 and extended to distinguish classes of groupoids associated with distributions in manifolds. These ideas are applied in Section 3 to define and interpret various cases of material groupoids.

\section{Groupoids and distributions}

2.1. Groupoids. Recall that a groupoid consists of a total set $\mathcal{Z}$, a base set $\mathcal{M}$, two (projection) surjective maps

$$
\alpha: \mathcal{Z} \rightarrow \mathcal{M} \text { and } \beta: \mathcal{Z} \rightarrow \mathcal{M}
$$

called, respectively, the source and the target maps, and a binary operation (composition) defined only for those ordered pairs $(y, z) \in \mathcal{Z} \times \mathcal{Z}$ such that

$$
\alpha(z)=\beta(y) .
$$

\footnotetext{
${ }^{1}$ By symmetry type we mean properties such as isotropy, transverse isotropy or orthotropy.
} 
This operation, indicated by the reverse apposition of the operands, must be associative, that is, $(x y) z=x(y z)$, whenever the products are defined. Moreover, at each point $m \in \mathcal{M}$, there exists an identity $\mathrm{id}_{m}$ such that $z \mathrm{id}_{m}=z$ whenever $\alpha(z)=m$, and $\operatorname{id}_{m} z=z$ whenever $\beta(z)=m$. Finally, for each $z \in \mathcal{Z}$ there exists a (unique) inverse $z^{-1}$ such that $z z^{-1}=\mathrm{id}_{\beta(z)}$ and $z^{-1} z=\mathrm{id}_{\alpha(z)}$.

It follows from this definition that to each ordered pair $(a, b)$ of elements of $\mathcal{M}$ one can associate a definite subset $\mathcal{Z}_{a b}$ of $\mathcal{Z}$, namely the subset $Z_{a b}=\{z \in \mathcal{Z} \mid$ $\beta(z)=b, \alpha(z)=a$ \}. It is clear that these subsets (some of which may be empty) are disjoint and that their union is equal to $\mathcal{Z}$. It is also clear that the various identities are elements of subsets of the form $\mathcal{Z}_{b b}$. It is not difficult to show that each set of the form $\mathcal{Z}_{b b}$ is actually a group. A useful way to think of a groupoid is as a collection of symbols $(a, b, c, \ldots \in \mathcal{M})$ and arrows $(x, y, z, \ldots \in \mathcal{Z})$ connecting some of them.

One can prove that if $\mathcal{Z}_{a b} \neq \varnothing$, then the groups $\mathcal{Z}_{a a}$ and $\mathcal{Z}_{b b}$ are conjugate, and the conjugation between them is achieved by any element $z$ of $\mathcal{Z}_{a b}$, namely,

$$
\mathcal{Z}_{b b}=z_{a a} z^{-1}
$$

Analogously, the set $\mathcal{Z}_{a b}$ is spanned completely by composing any one of its elements with $\mathcal{Z}_{a a}$ or with $\mathcal{Z}_{b b}$, that is,

$$
\mathcal{Z}_{a b}=z \mathcal{Z}_{a a}=\mathcal{Z}_{b b} z
$$

A groupoid is said to be transitive if for each pair of points $a, b \in \mathcal{M}$ there exists at least one element of the total set with $a$ and $b$ as the source and target points, respectively. In other words, a groupoid is transitive if, and only if, $\mathcal{Z}_{a b} \neq \varnothing$ $\forall(a, b) \in \mathcal{M} \times \mathcal{M}$. In a transitive groupoid all the local groups $\mathcal{Z}_{b b}$ are mutually conjugate. In this case, we can consider any of the local groups as the typical group of the transitive groupoid.

A groupoid is a topological groupoid if the total set $\mathcal{Z}$ and the base set $\mathcal{M}$ are topological manifolds and the projections $\alpha$ and $\beta$ are continuous, as are the operations of composition and of inverse. It follows from the definition that each of the sets $\mathcal{Z}_{b b}$ is a topological group. If $\mathcal{Z}$ and $\mathcal{M}$ are smooth manifolds and if both projections are surjective submersions and all operations are smooth, we obtain a Lie groupoid. ${ }^{2}$

2.2. Groupoids subordinate to a distribution. Let $\mathcal{C}$ be an $n$-dimensional manifold and let $0<k<n$ be an integer. A $k$-dimensional distribution $\mathcal{D}$ in $\mathcal{C}$ is a smooth assignment of a $k$-dimensional subspace $D_{c}$ of the tangent space $T_{c} \mathcal{C}$ to each point $c \in \mathcal{C}$. That the assignment is smooth means that each point $c \in \mathcal{C}$ has

\footnotetext{
${ }^{2}$ For a thorough treatment of Lie groupoids see [Mackenzie 1987] or [Mackenzie 2005].
} 
a neighbourhood within which there exist $k$ smooth linearly independent vector fields that span the subspaces of the distribution. In fact, a common way to specify a $k$-dimensional distribution is by providing $k$ linearly independent smooth vector fields on $\mathcal{C}$. A vector field $\boldsymbol{V}$ belongs to the distribution if, and only if, it is a linear combination of the vector fields defining it.

A groupoid $\alpha, \beta: \mathcal{Z} \rightarrow \mathcal{C}$ is said to be subordinate to a distribution $\mathcal{D}$ in $\mathcal{C}$ if every element $z \in \mathcal{Z}$ is a nonsingular linear map

$$
z: D_{c_{1}} \rightarrow D_{c_{2}}
$$

with $c_{1}, c_{2} \in \mathcal{C}$.

Given a distribution $\mathcal{D}$ in $\mathcal{C}$, all possible groupoids subordinate to the distribution are subsets (subgroupoids) of the transitive Lie groupoid $\mathcal{Z}_{\mathcal{D}}$ obtained by considering all the possible nonsingular linear maps between all subspaces $\mathcal{D}_{c}$ in the distribution.

A case of particular importance arises when the base manifold $\mathcal{C}$ is a fibre bundle $\pi: \mathcal{C} \rightarrow \mathcal{B}$ over an $m$-dimensional base manifold $\mathcal{B}$, with $m<n$. At each point $c \in \mathcal{C}$ the tangent space $T_{c} \mathcal{C}$ has a canonically defined vertical subspace $V_{c}$, which can be identified with the tangent space $T_{c} \mathcal{C}_{\pi(c)}$ to the fibre over $b=\pi(c)$ (that is, the set $\left.\pi^{-1}(\{b\}), b \in \mathcal{B}\right)$. A vector in $T_{c} \mathcal{C}$ belongs to the vertical subspace $V_{c}$ (or: is vertical) if, and only if, its projection by $\pi_{*}$ is the zero vector of $T_{\pi(c)} \mathcal{B}$. The existence of these vertical subspaces allows us to define the canonical $(n-m)$ dimensional vertical distribution $\mathcal{V}$ in the bundle $\mathcal{C}$. Accordingly, a groupoid $\mathcal{Z}$ subordinated to $\mathcal{V}$ will be called a vertical groupoid.

A vertical groupoid on a fibre bundle may or may not be transitive. An intermediate situation is worthy of consideration. We say that a vertical groupoid is fibrewise transitive if

$$
\pi\left(c_{1}\right)=\pi\left(c_{2}\right) \Longrightarrow \mathcal{Z}_{c_{1} c_{2}} \neq \varnothing
$$

A fibrewise transitive vertical groupoid is transitive if, and only if, for every pair $a, b \in \mathcal{B}$ there exists a pair $c_{a}, c_{b} \in \mathcal{C}$, with $\pi\left(c_{a}\right)=a$ and $\pi\left(c_{b}\right)=b$, such that $\mathcal{Z}_{c_{a} c_{b}} \neq \varnothing$. The truth of this assertion follows from the associative property of the groupoid composition.

2.3. Groupoids subordinate to an Ehresmann connection. In a fibre bundle $\pi$ : $\mathcal{C} \rightarrow \mathcal{B}$, if a vector in $T_{c} \mathcal{C}$ is not vertical, there is no canonical way to assign to it a vertical component. An Ehresmann connection provides this assignment.

Formally, an Ehresmann connection $\mathcal{H}$ consists of a smooth horizontal distribution in $\mathcal{C}$. This is a smooth assignment to each point $c \in \mathcal{C}$ of a subspace $H_{c} \subset T_{c} \mathcal{C}$ (called the horizontal subspace at $c$ ), of the same dimension as the base manifold $\mathcal{B}$, 


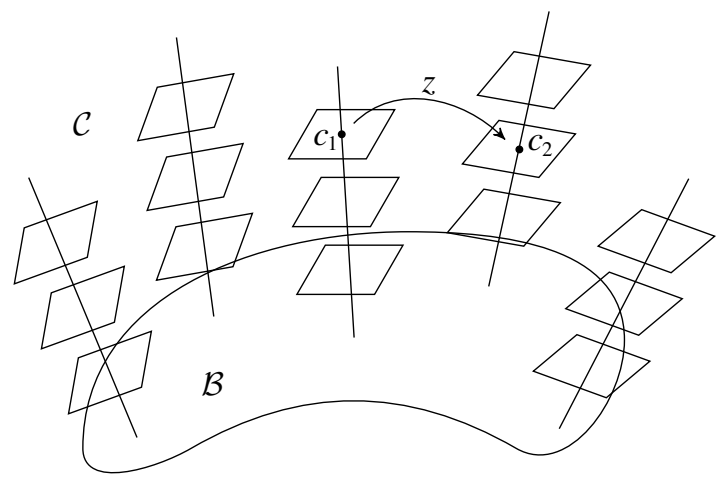

Figure 1. An element (arrow) in a horizontal groupoid.

such that

$$
T_{c} \mathcal{C}=H_{c} \oplus V_{c}
$$

In this equation, $\oplus$ denotes the direct sum of vector spaces. Each tangent vector $\boldsymbol{v} \in T_{c} \mathcal{C}$ is, accordingly, uniquely decomposable as the sum of a horizontal part $h(\boldsymbol{v})$ and a vertical part $v(v)$. A vector is horizontal if its vertical part vanishes. The only vector that is simultaneously horizontal and vertical is the zero vector.

Given a fibre bundle $\pi: \mathcal{C} \rightarrow \mathcal{B}$ endowed with an Ehresmann connection $\mathcal{H}$, we define a horizontal groupoid $\alpha, \beta: \mathcal{Z} \rightarrow \mathcal{C}$ as a groupoid whose elements $z \in \mathcal{Z}$ are nonsingular linear maps

$$
z: H_{c_{1}} \rightarrow H_{c_{2}}
$$

Notice that no a priori restriction is imposed on $c_{1}, c_{2} \in \mathcal{C}$, so that, in general, we may have $\pi\left(c_{1}\right) \neq \pi\left(c_{2}\right)$. Figure 1 illustrates this idea.

A horizontal groupoid is fibrewise transitive if

$$
\pi\left(c_{1}\right)=\pi\left(c_{2}\right) \Longrightarrow \mathcal{Z}_{c_{1} c_{2}} \neq \varnothing
$$

As in the case of a vertical groupoid, a fibrewise transitive horizontal groupoid is transitive if, and only if, for every pair $a, b \in \mathcal{B}$ there exists a pair $c_{a}, c_{b} \in \mathcal{C}$, with $\pi\left(c_{a}\right)=a$ and $\pi\left(c_{b}\right)=b$, such that $\mathcal{Z}_{c_{a} c_{b}} \neq \varnothing$.

Note. Given an Ehresmann connection, it is possible to define a particular isomorphism between all the horizontal spaces at points lying on one and the same fibre. These Christoffel isomorphisms, however, may or may not belong to a given horizontal groupoid defined on the bundle.

In a product bundle $\mathcal{C}=\mathcal{B} \times \mathcal{F}$ we can always define a canonical Ehresman connection in an obvious way. Moreover, as dictated by convenience in particular applications, the roles of the base manifold and the typical fibre can be interchanged. 


\section{Uniformity and evolution}

3.1. The body-time manifold. The concepts introduced in Section 2 can, in principle, be exploited for various applications in continuum mechanics. Material bodies with internal structure, for example, are themselves modeled as fibre bundles. Classical space-time can be regarded as an affine bundle over the real line. General relativity provides an opportunity to regard a history as a world tube with identifiable world lines. For our purposes, however, which aim at a unified picture of material uniformity and evolution, we will content ourselves with a body-time manifold consisting of the product $\mathcal{C}=\mathbb{R} \times \mathcal{B}$ of the time line $\mathbb{R}$ with an ordinary body $\mathcal{B}$. Moreover, adopting a fixed inertial observer, we identify space-time with the product bundle $\mathcal{S}=\mathbb{R} \times \mathbb{R}^{3}$. In this simple setting, both time and space are admittedly absolute, but the essence of the unified geometric picture is not greatly altered.

A history of the body can be regarded as a fibre-bundle morphism

$$
K: \mathcal{C} \rightarrow \mathcal{S}
$$

such that the map between the base manifolds is the identity. Thus, for every instant of time $t \in \mathbb{R}$, our morphism provides us with a map $\kappa_{t}: \mathcal{B} \rightarrow \mathbb{R}^{3}$, assumed to be an embedding, whose derivative at $X \in \mathcal{B}$ is a linear map

$$
F=F(X, t): T_{X} \mathcal{B} \rightarrow T_{\kappa_{t}(X)} \mathbb{R}^{3} \cong \mathbb{R}^{3},
$$

called the deformation gradient at $X \in \mathcal{B}$ at time $t$.

3.2. Constitutive considerations. The geometrical features of a material body described so far arise from the very nature of the body and of the physical space as continuous entities as well as from the kinematic manifestations of the former within the latter. An important feature of continuum mechanics is that the constitutive aspects of the material medium result in additional geometric structures. This material geometry arises essentially from a comparison of the material responses at different body points and at different instants of time. In view of the generality of the notion of groupoid and its ability to embrace both local and distant symmetries under the umbrella of a single mathematical entity, it is not unreasonable to expect that the material geometry alluded to above can be completely encapsulated within the compass of a single material groupoid.

We have just shown how any history of the body gives rise, by differentiation, to a collection of linear maps $F$ between the vertical subspaces of the body manifold and $\mathbb{R}^{3}$. In a simple or first-grade body, we assume that the constitutive response at each body point and at each instant of time can be completely encoded in one or more functions of $F$. These functions may be scalar-valued (such as the energy density of a solid) or take values in a space of tensors (such as the stress). Without much loss of generality, we will assume the case of a single scalar-valued 
function $\psi=\psi_{c}(F)$ for each point $c=(t, X) \in \mathcal{C}$. $^{3}$ We will assume all admissible constitutive functions $\psi_{c}(F)$ belong to a prespecified function space $\Psi$, such as $\Psi=C^{\infty}(\mathrm{GL}(3 ; \mathbb{R}))$.

A useful way to look at the constitutive response of a specific body-time manifold consists of considering the Cartesian product $\mathcal{C}_{\Psi}=\mathcal{C} \times \Psi$. The constitutive response is then a section $\sigma$ of $\mathcal{C}_{\Psi}$, namely a map

$$
\sigma: \mathcal{C} \rightarrow \mathcal{C}_{\Psi}
$$

such that $\mathrm{pr}_{1} \circ \sigma=\mathrm{id}_{\mathcal{C}}$, where $\mathrm{pr}_{1}$ denotes the first projection map in a product. In practice, this cross section is determined after choosing a particular reference configuration, that is, an identification of $\mathcal{B}$ with a domain in $\mathbb{R}^{3}$. We will soon see the influence of this choice on the resulting geometric entities. Before proceeding, however, we need to review the notion of action of a groupoid on a set.

\subsection{Action of a groupoid on a set.}

3.3.1. Group actions. For the sake of clarity, we will first review the idea of right (or left) action of a group on a set, as it is widely used in physical applications. Since a groupoid is, in some sense, a generalization of a group, it should not be surprising that the extended idea of groupoid action on a set can be conceived. This extension, however, is far from trivial.

If $\mathcal{G}$ is a group and $A$ is a set, we say that $\mathcal{G}$ acts on the right on $A$ if for each $g \in \mathcal{G}$ there is a map $R_{g}: A \rightarrow A$ such that (i) $R_{e}(a)=a$ for all $a \in A$, where $e$ is the group identity; (ii) $R_{g} \circ R_{h}=R_{h g}$ for all $g, h \in \mathcal{G}$. The order of the composition is the essential difference between a right and a left action. When there is no room for confusion, we also use the notation $a g$ for $R_{g}(a)$. With this notation, property (ii) neatly reads $a(h g)=(a h) g$.

It is not difficult to show that each of the maps $R_{g}$ is necessarily bijective. For this reason, these maps are also called transformations. Moreover, the inverse transformation is obtained as $\left(R_{g}\right)^{-1}=R_{g^{-1}}$. If we select a point $a$ in $A$ and follow its image $a g=R_{g}(a)$ as $g$ varies within $\mathcal{G}$, we obtain a subset of $A$ called the orbit through $a$, denoted by $a \mathcal{G}$. Orbits are disjoint subsets. The relation of "belonging to the same orbit" is an equivalence relation. The orbits themselves are the equivalence classes. An often useful concept is the quotient set, which is the set whose elements are the orbits of $A$.

The action of $\mathcal{G}$ on $A$ is said to be effective if the condition $R_{g}(a)=a$ for every $a \in A$ implies $g=e$. The action is free if $R_{g}(a)=a$ for some $a \in A$ implies $g=e$. Finally, the action is transitive if for every $a, b \in A$ there exists $g \in \mathcal{G}$ such that $R_{g}(a)=b$.

\footnotetext{
${ }^{3}$ The time dependence is often mediated by other variables which may obey additional evolution (constitutive) equations.
} 
As a pertinent example of a right group action, consider the following right action of the general linear group $\mathcal{G}=\mathrm{GL}(3 ; \mathbb{R})$ on the set $\Psi$ described above. An element of $\Psi$ is a possible constitutive equation $\psi=\psi(F)$ for a material point. Let $P \in \mathrm{GL}(3 ; \mathbb{R})$. We define

$$
R_{P}(\psi)=\psi(F P)
$$

Thus, the right action assigns to each constitutive equation another constitutive equation that, in the parlance of continuum mechanics, differs from the original one by the adoption of a different local reference. The orbit $\psi \mathcal{G}$, therefore, represents all the constitutive equations related in this way. From the physical standpoint, every orbit represents a different material, while the points in one orbit are different manifestations of the same constitutive law in different reference configurations. The symmetry group $\mathcal{G}_{\psi}$ of a constitutive equation $\psi$ is defined as the largest subgroup of $\mathcal{G}$ that leaves $\psi$ invariant. In other words,

$$
G \in \mathcal{G}_{\psi} \Longleftrightarrow R_{G}(\psi)=\psi .
$$

3.3.2. Groupoid actions. A (left) action of a groupoid $\alpha, \beta: \mathcal{Z} \rightarrow \mathcal{B}$ on a set $A$ consists of two maps. The first map,

$$
\rho: A \rightarrow \mathcal{B}
$$

is known as an anchor map, assumed to be surjective. To introduce the second map, representing the action itself, we consider first the subset $\mathcal{Z} * A$ of the Cartesian product $\mathcal{Z} \times A$ defined as

$$
\mathcal{Z} * A=\{(z, a) \in \mathcal{Z} \times A \mid \rho(a)=\alpha(z)\} .
$$

An action map $U$, given by

$$
U: \mathcal{Z} * A \rightarrow A
$$

must satisfy the following (rather expected) conditions:

(1) Consistency:

$$
\rho(U(z, a))=\beta(z) \quad \forall(z, a) \in \mathcal{Z} * A .
$$

(2) Composition:

$$
U(y z, a)=U(y, U(z, a))
$$

whenever the operations are defined.

(3) Unit:

$$
U\left(\operatorname{id}_{\rho(a)}, a\right)=a \quad \forall a \in A .
$$

These properties are schematically represented in Figure 2. 


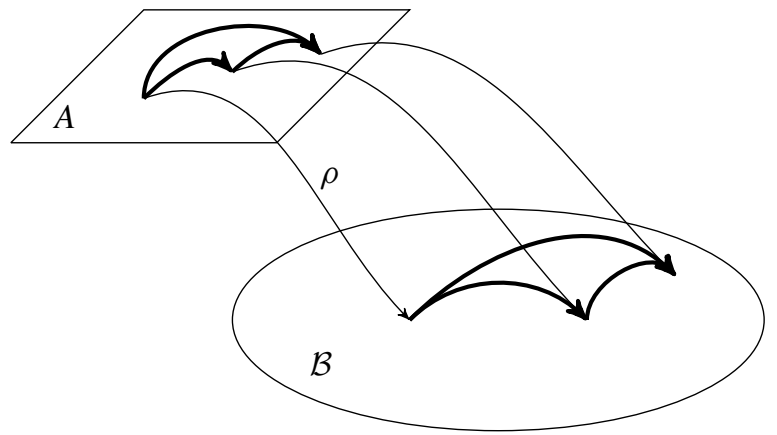

Figure 2. Left action of a groupoid $\alpha, \beta: \mathcal{Z} \rightarrow \mathcal{B}$ on a set $A$.

\subsection{The body-time material groupoids.}

3.4.1. Action of $\mathcal{Z}_{\mathcal{V}}$ on $\mathcal{C}_{\Psi}$. In Section 2.2 we introduced the canonical groupoid $\mathcal{Z}_{\mathcal{D}}$ subordinate to a distribution $\mathcal{D}$ defined as the collection of all possible nonsingular linear maps between all the subspaces in the distribution. We apply this notion to the case in which the manifold $\mathcal{C}$ is the body-time manifold and the distribution is identified with the vertical distribution $\mathcal{V}$. The groupoid $\mathcal{Z}_{\mathcal{V}}$ has a natural action on the set $\mathcal{C}_{\Psi}$ defined in Section 3.2. The anchor map is simply the trivial bundle projection in $\mathcal{C}_{\Psi}$. The action $U(z,(c, \psi))$ of an element $z \in \mathcal{Z}_{\mathcal{V}}$ on the pair $(c, \psi) \in \mathcal{C}_{\Psi}$ such that $\alpha(z)=c$ is defined as

$$
U(z,(c, \psi))=\left(\beta(z), R_{P(z)}(\psi)\right),
$$

where $R$ is the right group action defined in (11) and $P(z)$ is the matrix associated with the element $z$ in the vertical groupoid $\mathcal{Z}_{\mathcal{V}}$. Note the apparent disagreement between the right character of the group action and the left action of the groupoid.

3.4.2. The material groupoid. A particular material response, as we have seen, is a particular cross section $\sigma$ of the product bundle $\mathcal{C}_{\Psi}$. Consider a subgroupoid $\mathcal{W}$ of $\mathcal{Z}_{\mathcal{V}}$. We can certainly restrict the action $U$ to $\mathcal{W}$. The constitutive section $\sigma$ may or may not be invariant under the action $U$ of $\mathcal{W}$. We define the material groupoid of a given body-time manifold with constitutive response $\sigma$ as the largest subgroupoid $\mathcal{W}$ of $\mathcal{Z}_{\mathcal{V}}$ that leaves $\sigma$ invariant under the action $U$. This definition makes sense because, on the one hand, the disjoint subgroupoid of the unit maps is always available (so that there is no danger that the material groupoid will be empty) and, on the other hand, given two subgroupoids with the desired property, the subgroupoid generated by their union also enjoys that property.

A graphical way to visualize the material groupoid consists of drawing an arrow between each pair of points $c_{1}$ and $c_{2}$ in the body-time manifold $\mathcal{C}$ for every material isomorphism $P$ between $c_{1}$ and $c_{2}$. The collection of all arrows thus drawn constitutes the material groupoid. The groupoid is transitive if all point pairs are 
arrowwise connected. The typical group of a transitive material groupoid is the symmetry group of any one of the body points.

3.4.3. The material-type groupoid. Within the set $\Psi$ we can introduce an equivalence relation $\sim$ as follows.

$$
\psi_{1} \sim \psi_{2} \Longleftrightarrow \exists H \in \operatorname{GL}(3 ; \mathbb{R}) \mid \mathcal{G}_{\psi_{1}}=H \mathcal{G}_{\psi_{2}} H^{-1} .
$$

In other words, two constitutive equations are -equivalent if, and only if, their respective symmetry groups are mutually conjugate. Define the quotient set

$$
\Psi^{\sim}=\Psi / \sim
$$

The elements of the quotient set are equivalence classes $\psi^{\sim}$. Physically, two constitutive equations are in the same equivalence class if they represent the same type of material (e.g., isotropic, transversely isotropic, orthotropic).

The groupoid $\mathcal{Z}_{\mathcal{V}}$ acts on $\mathcal{C} \times \Psi^{\sim}$ in an obvious way. Moreover, the constitutive section $\sigma$ induces uniquely a section $\sigma^{\sim}: \mathcal{C} \rightarrow \mathcal{C} \times \Psi^{\sim}$ via the map $\sim: \psi \rightarrow \psi^{\sim}$. We define the material-type groupoid as the largest subgroupoid of $\mathcal{Z}_{\mathcal{V}}$ that leaves the section $\sigma^{\sim}$ invariant.

As we have suggested for the case of the material groupoid, we may visualize the material-type groupoid by drawing an arrow between each pair of points $c_{1}$ and $c_{2}$ in the body-time manifold $\mathcal{C}$ for every conjugation $H$ between the symmetry groups $\mathcal{G}_{c_{1}}$ and $\mathcal{G}_{c_{2}}$. The collection of all arrows thus drawn constitutes the materialtype groupoid. Clearly, the material groupoid is a subgroupoid of the materialtype groupoid, since materially isomorphic points have conjugate symmetry groups. The typical group of a transitive material-type groupoid is given by the normalizer of the symmetry group of any of the body points.

\subsection{Physical interpretation.}

3.5.1. The body-time material groupoid. The terminology used to describe various kinds of distant symmetries, relevant to, among other areas, the theories of continuous distributions of defects and the theories of biological growth and remodeling, is not completely standardized. We thus start by fixing a terminological scheme to interpret the differential-geometric picture in physically meaningful terms.

In the introduction, we referred to the concepts of material isomorphism and uniformity as they pertain to the purely spatial (as opposed to temporal) component of the body-time description. Two points $a$ and $b$ of a material body $\mathcal{B}$ are materially isomorphic if there exists a nonsingular linear map $P: T_{a} \mathcal{B} \rightarrow T_{b} \mathcal{B}$ such that their respective constitutive functions, $\psi_{a}(F)$ and $\psi_{b}(F)$, are related by the identity

$$
\psi_{b}(F)=\psi_{a}(F P)
$$


for all deformation gradients $F$. Points $a$ and $b$ are, therefore, made of the same material. Within this context, a local material symmetry $G$ at point $a \in \mathcal{B}$ can be regarded as a material endomorphism $G: T_{a} \mathcal{B} \rightarrow T_{a} \mathcal{B}$. The collection of symmetries at $a$ forms a group $\mathcal{G}_{a}$. If $a$ and $b$ are materially isomorphic, their respective symmetry groups are conjugate. More specifically, if $P$ is a material isomorphism, then $\mathcal{G}_{b}=P \mathcal{G}_{a} P^{-1}$. Conversely, if $a$ and $b$ are materially isomorphic, the cardinality of the set $\mathcal{P}$ of all possible material isomorphisms between $a$ and $b$ is the same as the cardinality of the respective (conjugate) symmetry groups. In fact, $\mathcal{P}$ can be generated from a given $P$ according to the formula

$$
\mathcal{P}=P \mathcal{G}_{a}=\mathcal{G}_{b} P=\mathcal{G}_{b} P \mathcal{G}_{a}
$$

A body is called materially uniform if all of its points are materially isomorphic. Since material isomorphism is an equivalence relation, a body is materially uniform if and only if all its points are materially isomorphic to a fixed reference body point.

The temporal counterpart of uniformity is a special kind of material evolution, whereby a material point remains materially isomorphic to a reference material point with the passage of time. This special kind of material evolution is common in the realm of biological tissues, with their natural tendency to adapt to their changing environments. A classical example is Wolff's law of trabecular bone, whose trabeculae are thought to change their orientations to follow the principal directions of stress. The fact that the material remains materially isomorphic to its initial state does not preclude the possibility of growth and resorption, whereby material of the same kind is added or removed volumetrically to the material neighbourhood. But material isomorphism does preclude the transformation of the underlying material in terms of variation of its intrinsic material properties and chemical composition. In a simplified model, we may imagine a constitutive response idealized as an elastic spring with a characteristic rest length and a given stiffness constant. Material isomorphism would imply that, while the rest length may change in time, the stiffness constant must remain unchanged. We call this special type of material evolution remodeling. Any other kind of evolution we call aging. We will later identify a particular kind of aging as worthy of further attention.

If we consider a transitive body-time material groupoid, its physical meaning is a body that is initially materially uniform and that evolves by pure remodeling (with no aging). In particular, it remains always materially uniform. Classical plastic evolution belongs to this material class and so does the model of tissue growth pioneered in [Rodriguez et al. 1994].

Assume, on the other hand, that the material groupoid is only fibrewise transitive, a concept introduced in Section 2.2. The meaning of this situation is that, while the body is materially uniform at all times, there is a process of aging taking place at all points at the same pace. In constitutive terms, this will be the case if the 
dependence of the constitutive equation $\psi=\psi(F ; t, X)$ is expressed explicitly in terms of the observer time $t$, rather than mediated by some internal variable subject to evolution conditions. In other words, the material degradation takes place by the mere passage of time, without any coupling to other observable phenomena (such as the state of stress).

Since the body-time manifold has been defined as a Cartesian product $\mathbb{R} \times \mathcal{B}$, we may exchange the role of the base and the typical fibre and consider instead the product manifold $\mathcal{B} \times \mathbb{R}$. In this product bundle, we have a natural horizontal distribution (Ehresmann connection) induced by the constant sections. The material groupoid can now be regarded, in the terminology of Section 2.3, as a horizontal groupoid. If this new material groupoid happens to be fibrewise transitive, we obtain the representation of a body that, without necessarily being materially uniform, evolves by pure remodeling, without aging. Notice that under these conditions if the body is initially nonuniform, it will never attain uniformity.

3.5.2. The body-time material-type groupoid. We want to explore now the physical meaning of the material-type groupoid. Recall that the "arrows" of this groupoid represent only conjugation maps between the material symmetry groups of the source and target points. Put differently, the elements of this groupoid are not sensitive to any constitutive property, except the symmetries of the constitutive law. If the material-type groupoid is transitive, all that this implies is that the different material points are of the same symmetry type (isotropic, say). For all we know, part of the body may be made of cement and the rest of rubber. Moreover, as time goes on, the cement may be undergoing a process of curing and change its elastic properties. A functionally graded material obtained by varying the relative concentration of the components of a mixture belongs to the same category, even if the components undergo chemical reactions, as long as the symmetry type is preserved.

If the material-type groupoid is only fibrewise transitive, we have a phenomenon of morphogenesis or symmetry breaking. In this instance, all the body points undergo a change of symmetry type simultaneously. In solid materials, where the collection of all possible symmetry groups is countable, this transition is, of necessity, abrupt. In some cases of phase transition these changes may be directly observable with the naked eye and be manifest as pattern formations. Reversing the role of the base and the fibre manifolds, a fibrewise transitive material-type groupoid represents a body with initially demarcated portions obeying different symmetry types and remaining so with the passage of time.

\section{References}

[Epstein and de León 2000] M. Epstein and M. de León, "Homogeneity without uniformity: towards a mathematical theory of functionally graded materials", Internat. J. Solids Structures 37:51 (2000), 7577-7591. 
[Epstein and Elżanowski 2007] M. Epstein and M. Elżanowski, Material inhomogeneities and their evolution: a geometric approach, Springer, Berlin, 2007.

[Mackenzie 1987] K. Mackenzie, Lie groupoids and Lie algebroids in differential geometry, London Mathematical Society Lecture Note Series 124, Cambridge University Press, 1987.

[Mackenzie 2005] K. C. H. Mackenzie, General theory of Lie groupoids and Lie algebroids, London Mathematical Society Lecture Note Series 213, Cambridge University Press, 2005.

[Noll 1967/68] W. Noll, "Materially uniform simple bodies with inhomogeneities", Arch. Rational Mech. Anal. 27 (1967/68), 1-32.

[Rodriguez et al. 1994] E. K. Rodriguez, A. Hoger, and A. D. McCulloch, "Stress-dependent finite growth in soft elastic tissues", J. Biomech. 27:4 (1994), 455-467.

[Turing 1952] A. M. Turing, "The chemical basis of morphogenesis", Philos. Trans. Roy. Soc. London Ser. B 237:641 (1952), 37-72.

[Wang and Bloom 1973/74] C. C. Wang and F. Bloom, "Material uniformity and inhomogeneity in anelastic bodies", Arch. Rational Mech. Anal. 53 (1973/74), 246-276.

[Weinstein 1996] A. Weinstein, "Groupoids: unifying internal and external symmetry. A tour through some examples”, Notices Amer. Math. Soc. 43:7 (1996), 744-752.

Received 25 Jul 2015. Revised 9 Dec 2015. Accepted 6 Feb 2016.

MARCELO EPSTEIN: mepstein@ucalgary.ca

Department of Mechanical and Manufacturing Engineering, University of Calgary, 2500 University Drive NW, Calgary, AB T2N 1N4, Canada

MANUEL DE LEÓN: mdeleon@icmat.es Instituto de Ciencias Matemáticas, CSIC-UAM-UC3M-UCM, Nicolás Cabrera, 13-15, 28049 Madrid, Spain 